V.K. Beliaev, H.N. Panenko

\title{
DETERMINATION OF INSULATION PARAMETERS OF CURRENT TRANSFORMERS AT MULTIPLE MEASUREMENTS IN MONITORING SYSTEMS UNDER WORKING VOLTAGE
}

Features of the data processing procedures of multiple measurements of the dielectric loss tangent of the high-voltage insulation, which are based on the comparison method, were considered. Three procedures were compared: the one procedure uses an assignment of a particular standard object for comparison, and two procedures don't use assignments of the special standard objects. Expressions of methodical errors of studied procedures were obtained. Analysis and calculations, which use the obtained expressions were made. Evaluations showed that an influence of a change of the tangent of dielectric loss of one from the monitoring objects on results of estimation of the tangents of other monitoring objects is the distinctive feature of the procedures without assignment of the standard object. Errors of these procedures more than errors of the traditional procedure, with assignment of the standard object. The study demonstrated that procedures without assignment of standard objects lead to difficultly predictable methodological errors, which hinder to estimate the actual value of the loss tangent and to estimate outcomes of the monitoring, by means comparing with allowable values. Moreover, the decrease of estimations of loss tangents of all objects, as a result of occurrence of at least one the object with strongly bad parameters, hides occurrence of other unsuccessful objects with smaller deviations and makes difficult recognition of such deviations at early stages. Improvements in processing techniques which reduce earlier specified errors and reduce the amount of measurements are proposed. The study results are useful in monitoring and diagnostic of basic insulation of current transformers or high-voltage bushings. References 8 , tables 1 , figures 5 .

Key words: high voltage insulation, diagnostics, data processing procedure, processing procedure error, dielectric loss tangent, current transformer.

Рассмотрены особенности методик обработки результатов множественных измерений тангенса угла диэлектрических потерь высоковольтной изоляции, основанных на методе сравнения. Проанализированы известные методики определения тангенса потерь с выделением отдельного эталонного объекта и без выделения такого эталона. Получены выражения для определения методических погрешностей. Проведено сравнение и показано, что применение методик обработки без выделения отдельного эталонного объекта может приводить к существенным сложностям в оценке результатов контроля изоляции. Предложены усовериенствования методик обработки, уменьшающие указанные погрешности методик и трудоемкость измерений. Библ. 8, табл. 1, рис. 5.

Ключевые слова: высоковольтная изоляция, диагностика, методика обработки, погрешность, тангенс угла диэлектрических потерь, трансформатор тока.

Introduction. The power companies of Ukraine introduced technologies of condition monitoring of highvoltage insulation of measuring current transformers without decommissioning (under operating voltage) [1-5]. At present, as the main method of such control for oilfilled current transformers (insulation of capacitor type) should be considered differential control in which among all tested current transformers (hereinafter referred to as objects of control - OC) of one phase they select «reference» having the best condition of the insulation, and others' state is evaluated by the change of dielectric loss tangent with respect to the selected reference $[3,6]$. We can select the technologies of periodic and continuous monitoring which differ, in fact, by the frequency of measurement of the insulation of objects of control (OC) which is a consequence of the degree of automation of the measurement process $[3,5]$. Similar tools can be used in both technologies (bridge, vector meters) and measuring methods.

To increase the efficiency of control technology it is proposed [2, 5] to use multiple measurements during which it is assumed that each OC is alternately used as a reference for all other controlled OC (without determination of separate reference object). Indicated approach eliminates the need for special control and awkward shifts of reference objects. These methods of measurements results treatment differ from the processing technique at selection of a reference by using various kinds of averages over all the results that will naturally lead to reduction of influence of random variations caused by, for example, short-term noises. At the same time, in [7] it is indicated on the possibility of occurrence at such treatment of additional errors not arise at traditional measurements processing with special release of the reference object.

The goal of the work is definition of error of techniques of results treatment of multiple measurements of the loss tangent of the insulation without selection of the reference object, the development of methods to reduce the error.

Fundamentals of methods of processing the results of measurements of loss tangent of insulation. A basis of differential control is a bridge method of

(C) V.K. Beliaev, H.N. Panenko 
comparison with a reference (standard) [3, 6]. Using AC bridge (recently high-precision measuring vector meters, for example [1] are used), they measure by tangent $\left(\operatorname{tg} \delta_{m e g}\right)$ of the difference of dielectric loss angles of OC $\left(\delta_{o k}\right)$ connected to the input $\langle\mathrm{X} »$ and angle of reference loss $\left(\delta_{e}\right)$ connected to the input «0», so that (because of the smallness of the actual loss angles the tangent of the difference from the difference of tangents can be ignored):

$$
\operatorname{tg} \delta_{o k}=\operatorname{tg} \delta_{m e g}+\operatorname{tg} \delta_{e} .
$$

The problem is that the actual value of the loss tangent of the reference object used as the base for counting, during the measurement (control) is unknown, and instead of it we use the approximate values that is a source of systematic errors - the calculated tangent differs from the actual OC loss tangent.

In the case of measuring a plurality of objects under operating voltage for one phase $\mathrm{OC}$ at any time $k$, we write the formula (1) as follows (for simplicity hereinafter instead $\operatorname{tg} \delta$ we write $\delta$ ):

$$
\delta_{j(k)}=\partial \delta_{i j(k)}+\delta_{i(k)}, \text { or } \partial \delta_{i j(k)}=\delta_{j(k)}-\delta_{i(k)},
$$

where $\delta_{j(k)}$ is the loss angle tangent of the $j$-th OC; $\partial \delta_{i j(k)}$ is the tangent of the differential angle at the measurement for the $j$-th OC at the $i$-th standard (the first index is for standard, the second one is for the tested OC). Here, according to [3], the measured tangent of OC loss of the angle difference and the standard is called as tangent of the differential angle.

At the differential control is determined not the tangent of the OC differential angle is determined but its increment $\left(\Delta \delta_{i j}\right)$ relative to baseline values - at the beginning of the control $[3,6]$. So, at the measurement at time $k$ for the $j$-th $\mathrm{OC}$ at the $i$-th reference (one pair measurement), the increment of tangent of the differential angle:

$$
\Delta \delta_{i j(k)}=\partial \delta_{i j(k)}-\partial \delta_{i j(0)}=-\Delta \delta_{j i(k)},
$$

where $\partial \delta_{i j(0)}$ is the initial value of the tangent of the differential angle measured at the taking of objects in the control.

According to the procedure 1 described in Standard COУ-H MПЕ [3], the measurements are carried out with the selected reference OC ( $i$ is the reference index). Using the obtained according to (3) increment, they determine the current estimated value of the tangent of the checked OC $[3,6]$ :

$$
\delta p_{j(k)}=\Delta \delta_{i j(k)}+\delta_{j(0)},
$$

where $\delta_{j(0)}$ is the initial value of the tangent for the $j$-th OC determined at the taking of objects in the control.

For the reference OC:

$$
\delta p_{i(k)}=-\Delta \delta_{i j(k)}+\delta_{i(0)} .
$$

The obtained values are compared with the permissible values of loss angle tangent $(0.005 \ldots$.. 0.008) normalized in [3] (rejection criteria).
Substituting the definition (3) in the formula (4), after transformations we obtain the following expression for determining the calculated value of tangent:

$$
\delta p_{j(k)}=\partial \delta_{i j(k)}+\delta_{i(0)} .
$$

Comparing (6) with the exact expression (2) for the actual value of OC tangent we see that in the procedure 1 at determining the calculated tangent it is proposed instead of unknown during measurement value of the reference OC tangent $\delta_{i(k)}$ to use the value of objects in the control determined at the taking of objects in the control $\delta_{i(0)}$. The resulting calculated value of the tangent of the loss angle $\delta p_{j(k)}$ (which is compared with the permissible norm) will differ from the actual $\delta_{j(k)}$ to the value of increment of the tangent of the reference object tangent for the time from the start of control.

At mass monitoring of $\mathrm{OC}$ insulation on the outdoor switchgear, there are certain inconveniences with the choice and the control of the state of the reference $\mathrm{OK}$, and when selection one standard for all OC phases (the most convenient option), formula (5) to assess the standard OC becomes unacceptable [5]. Indeed, for each checked OC without changing the reference we obtain individual different result from the others for $\Delta \delta_{i j(k)}$ and as a consequence - different values of calculated tangent $\delta p_{i(k)}$ for the same $i$-th reference.

To be able to control the reference $\mathrm{OC}$, in the Dniprovska Power Grid [5] it was proposed instead of one particular measured value $\Delta \delta_{i j(k)}$ to use in (5) the average increment for all $N$ received values for the controlled objects on the phase:

$$
\delta p_{i(k)}=-\frac{1}{N-1} \sum_{j \neq i}^{N}\left(\Delta \delta_{i j(k)}\right)+\delta_{i(0)},
$$

where $i$ is the reference index.

Processing techniques without selection of the individual reference. In cases of an automated continuous or periodic monitoring of current transformers, several methods (algorithms) for determining the calculated tangent based on conducting plurality of measurements without selection of individual OC reference are proposed $[2,7]$. In these measurements each OC alternately serves as a reference for the other checked OC of the same phases. As each OC passes measurements in pair with another one, the total number of measurements increases considerably - as the square of OC number.

Method 2 used AT nine substations OF THE Donbass power system [5] At the control of $N$ objects on the phase it is proposed in determining the current value of each OC calculated tangent instead of formulas (4) and (5) using the following expressions constructed by analogy with formula (7):

$$
\delta p_{i(k)}=-\Delta \delta_{i a v(k)}+\delta_{i(0)}, \Delta \delta_{i a v(k)}=\frac{1}{N-1} \sum_{j \neq i}^{N} \Delta \delta_{i j(k)}
$$


where the index iav indicated the value average for all $\mathrm{OC}$ (excluding the $i$-th one.

We merge (8) in one expression and substitute the definition (3):

$$
\begin{aligned}
& \delta p_{i(k)}=-\frac{1}{N-1} \sum_{j \neq i}^{N} \Delta \delta_{i j(k)}+\delta_{i(0)}= \\
& =\frac{1}{N-1} \sum_{j \neq i}^{N}\left(-\partial \delta_{i j(k)}+\delta_{j(0)}\right)=-\partial \delta_{i a v(k)}+\delta_{i a v(0)} .
\end{aligned}
$$

The obtained expression shows (cf. (6)) that, according to this method, the calculated value of the loss tangent is the average of all paired results (each OC with everyone, except as with itself) carried out according to the method 1.

Procedure 3 proposed in [2] for use in the continuous control system at $330 \mathrm{kV}$ substation of the Dnepr power system. According to [2], at the control of $N$ objects eponymous phase is first determined by the intermediate values of tangent $\left(\delta 1_{i j(k)}\right)$ for each $i$-th OC at paired measurements with different standards $\mathrm{j}$ by the formula similar to (6), where instead of the tangent of the reference in the beginning of the control, use the calculated value of the tangent of the $i$-th OC obtained in previous measurements. To determine the final estimated value for the $i$-th OC taken the average of all intermediate values for this $\mathrm{OC}$ at different references $j$. The described in the formula expression can be written as follows:

$$
\delta 1_{j i(k)}=\delta p_{j(k-1)}-\partial \delta_{i j(k)}, \delta p_{i(k)}=\frac{1}{N-1} \sum_{j \neq i}^{N} \delta 1_{j i(k)}
$$

We write last formulae as follow:

$$
\begin{aligned}
& \delta p_{i(k)}=\frac{1}{N-1} \sum_{j \neq i}^{N}\left(-\partial \delta_{i j(k)}+\delta p_{j(k-1)}\right)= \\
& =-\partial \delta_{i a v(k)}+\delta p_{i a v(k-1)} .
\end{aligned}
$$

Comparing the expressions (11) and (9) we see that the results of the processing algorithms to determine the estimated value of the tangent of the last two methods, without isolation of the individual reference, differ only in the values taken for the baseline from which the increments are counted. In the second method of the base taken the average of all values of the tangents at the OC to take control in the third uses an average of all the values calculated in the previous measurement. Both techniques are used an average of the measured values of the tangent of the differential angle.

Comparison of methods' of errors. Of interest is the analysis of systematic errors arising as a result of the loss tangent calculation formulas given above, instead of the exact formula (2). Under the methodological error of the specific techniques mean difference between the calculated values of OC tangent, obtained by treatment of the appropriate expression of its real value:

$$
D_{j(k)}=\delta p_{j(k)}-\delta_{j(k)}
$$

Presenting each technique as a separate model to determine the output value (the estimated tangent), this error can be seen as error adequacy model showing the lowest possible error when using the model-technique [8]. The resulting expression for determining $b_{i(k)}$ of all the considered methods are given in the Table 1. For the methods of quality characteristics it is advisable to carry out sensitivity analysis, identifying the corresponding coefficients of sensitivity (influence) that bind each change of the input variable with the resulting change in the output. The table gives the expressions for the coefficients $b_{i(k)}$ characterizing the sensitivity of the resulting changes in the estimated value of tangent $j \mathrm{OC}$ at $k$ time $\varepsilon \delta p_{j(k)}$ to change in the actual value of the tangent of each $i$ OC $\varepsilon \delta_{i(k)}$ :

$\varepsilon \delta p_{j(k)}=\sum_{k 1=1}^{k}\left(\sum_{i} b_{i(k 1)} \varepsilon \delta_{i(k 1)}\right)+\sum_{i} b_{i(0)} \varepsilon \delta_{i(0)}, i, j=1 \ldots N$.

Here the general expression of connection is presented. Obviously, the components associated with the timing of 1 to $k-1$ appear only when using the method 3 . Assuming little change input values, sensitivity coefficients were determined based on derived respective functional connections of the output variable with input [8]. The coefficients of the last part of the above expression $(k=0)$ is characterized by sensitivity to errors taking of objects in the control.

The presented in Table 1 expressions show that the greatest impact on the estimated result for any checked OC values have the latest measurements on the same OC $\left(b_{j(k)}=1\right)$. Values of other OC tangents no effect on the inspected result in the case of $\mathrm{OK}$ method 1 , but can significantly affect the use of other techniques. This effect is inversely proportional to the number of OC.

Negative signs of influence factors $b_{i \neq i(k)}$ indicate that any increase in the value of the tangent at any OC would reduce the calculated values on other OC tangents.

For example, we suppose that the change occurred at one («damaged») OC. Let up to the $k$-th point in time no changes have occurred. The $k$-th time at the $m$-th OC abruptly changed by the amount of loss tangent $\varepsilon \delta_{m(k)}=\delta_{m(k)}-\delta_{m(k-1)}=d$, the remaining OC changes were not tangent $\left(\varepsilon \delta_{j \neq m(k)}=0\right)$ and at subsequent times tangent values at all OC is not changed $\left(\delta_{i(k+n)}=\delta_{i(k+1)}=\delta_{i(k)}\right)$ for all $i, n$. In accordance with the values of the coefficients of influence by (12) we change tangent calculation and by the expressions in Table 1.

We define $D_{j(k)}$ errors:

- By method 1: for «damaged» OC

$\varepsilon \delta p_{m(k)}=d, D_{j(k)}=0$; for other OC $\varepsilon \delta p_{j \neq m(k)}=0, D_{j \neq m(k)}=0$.

- By method 2: for «damaged» OC

$\varepsilon \delta p_{m(k)}=d, D_{j(k)}=0$; for other OC $\varepsilon \delta p_{j \neq m(k)}=-d /(N-1)$, $D_{j \neq m(k)}=-d /(N-1)$.

- By method 3: for «damaged» OC at time $k$ : $\varepsilon \delta p_{m(k)}=d, D_{j(k)}=0$; at the next time $\varepsilon \delta p_{j \neq m(k+1)}=-d /(N-1)$, $D_{j \neq m(k+1)}=-d /(N-1)$; for other OC at time $k: \varepsilon \delta p_{j \neq m(k)}=$ $=-d /(N-1), \quad D_{j \neq m(k)}=-d /(N-1) ; \quad$ at the next time $\varepsilon \delta p_{j \neq m(k+1)}=-d /(N-1)^{2}, D_{j \neq m(k+1)}=-d(N-2) /(N-1)^{2}$. 
As we can see, using methods 2 and 3, the calculated values differ from the actual tangent of its values $\left(\varepsilon \delta p_{j(k)} \neq\right.$ $\left.\varepsilon \delta_{j(k)}, D_{j(k)} \neq 0\right)$ to appear for «healthy» OC. When using 3 methods calculated tangents differ from the actual not only in «healthy», but also in «damaged» $\mathrm{OC}$ - not at the moment of change, but starting from the next measurement.

Table 1

Expressions for the considered methods' errors and sensitivity (influence) coefficients

\begin{tabular}{|c|c|c|}
\hline $\begin{array}{c}\text { Method No., } \\
\text { Calculation formula }\end{array}$ & Error $D_{j(k)}:$ & Sensitivity coefficient \\
\hline $1,(4), i$ - reference & $-\left(\delta_{i(k)}-\delta_{i(0)}\right)$ & $b_{j(k)}=1, b_{i(k)}=-1, b_{i(0)}=1$ \\
\hline $2,(9)$ & $-\frac{1}{N-1} \sum_{i \neq j}^{N}\left(\delta_{i(k)}-\delta_{i(0)}\right)$ & $b_{j(k)}=1, b_{i \neq j(k)}=-1 /(N-1), b_{i \neq j(0)}=1 /(N-1)$ \\
\hline $3,(11)$ & $-\frac{1}{N-1} \sum_{i \neq j}^{N}\left(\delta_{i(k)}-\delta p_{i(k-1)}\right)$ & $b_{j(k)}=1, b_{i \neq j(k)}=-1 /(N-1), b_{j(k-1)}=-1 /(N-1)$, \\
& $b_{i \neq j(k-1)}=1 /(N-1)^{2}, \ldots, b_{j(0)} \rightarrow 0$ \\
\hline $4,(13)$ & $-\frac{1}{N m-1} \sum_{i \neq j}^{N m}\left(\delta_{i(k)}-\delta_{i(0)}\right)$ & $b_{j(k)}=1, b_{i \neq j(k)}=-1 /(N m-1), b_{i \neq j(0)}=1 /(N m-1)$. \\
\hline
\end{tabular}

In actual control of a plurality of current transformers may be situations where the aging of the insulation at the same time gradually increasing the tangents at several objects at different speeds. Upon reaching the critical normalized values of tangents at one of $\mathrm{OK}$ decision on further testing or withdrawal from service. [3] In such cases, the effect of varying tangents of one $\mathrm{OK}$ on other using methods 2 or 3 difficult to assess the condition of the insulation.

For example, we consider a model situation of control of several OC $(N=6$, in [5] it is recommended to use no more than 6 OC due to the laborious measurements). Fig. 1 shows an idealized picture of the actual values change tangents insulation of OC (initial values from 0.0015 to 0.001 ) during the operation at a constant rate - from measurement to measurement ( $k$ is time, measuring index). For OC No. 2 strong change leads to achievement on the 20th step of monitoring a large critical value $(0.005)$, in the OC No. 3,4 growth weaker, for OC No. 1 poorly discernible rise (20 times less than that for No. 2) ,for two of the remaining changes are absent.

Fig. 2-4 show graphs of the calculated tangents, respectively defined by the formulas (4), (9) and (11) corresponding to the methods 1,2 and 3 . When using the method 1 (Fig. 2) as the reference OK No. 1 is adopted with a slight, but not with the smallest change that occurs in practice.

Accordingly, the calculated values of the tangents at the monitored objects smaller than actual (Fig. 1) to the value of growth of the reference OC tangent. For OC with the same with the real tangents will fix them a slight decrease (for the same value). Status of the reference OK by this method is not evaluated.

Fig. 2 also shows the calculated reference OK No. 1 tangents defined by the formula (7) (graph as 1a is indicated), which show that the evaluation of the situation in the considered yields a significant underestimation of the tangent, is the same as using the method 2 (see Fig. 3 below).

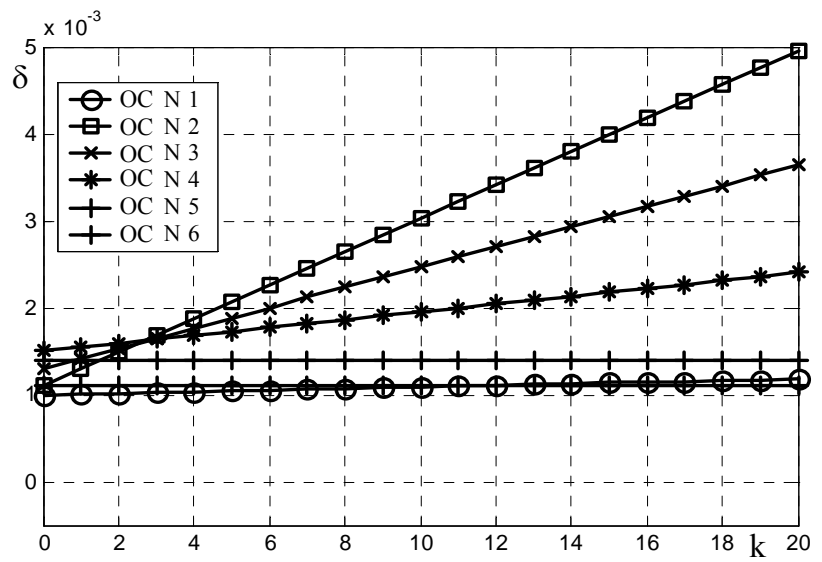

Fig. 1. Changes of real values of OC insulation loss tangents

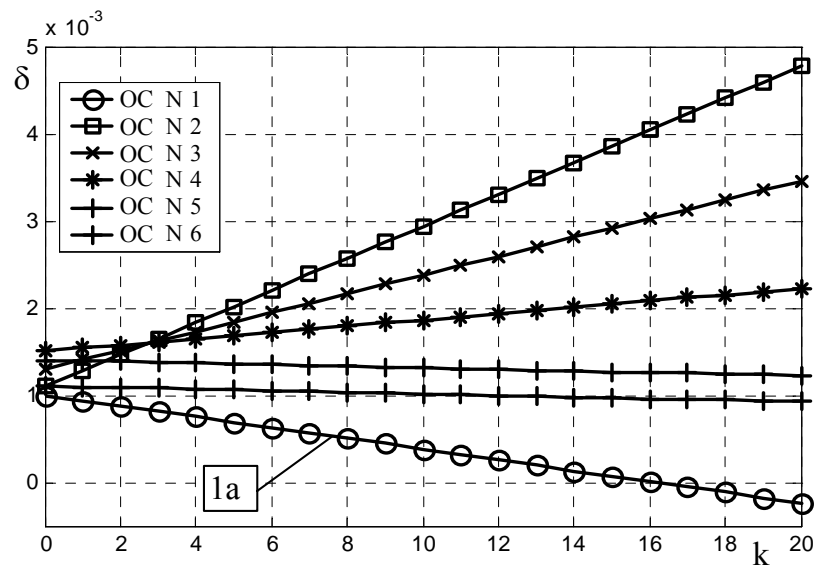

Fig. 2. Changes of calculated values of OC insulation loss tangents determined by method 1 
The graphs in Fig. 3 and 4 clearly show a strong change OK tangent tan calculated values but significantly different from the actual values. It is also readily determined by the relative state of isolation of objects of control, but to determine how big the actual values of tangents (which is necessary to identify the values exceeds the regulation) and how fast they are growing, it is difficult. To recognize errors in such an object with a small increase of the tangent (the initial stages of development defects) is a hardly feasible task.

It should be noted that when using the method of 3 difference between the calculated values of the tangents from the actual more than method 2. Method 1 is easier to have the predicted error (determined by the state of the reference OC). Given that the selected object as a reference in the best condition, an error procedure is one less than the methods 2 and 3.

The difficulty of assessing the real value of tangents and their trends according to methods 2 and 3 caused by $D_{j(k)}$ error dependence $(k)$ not only on the number of controllable OC, but the total gain change reality tangents (see expression in Table 1) which is unknown value in advance. The real situation is complicated by the uneven growth of losses, the temperature dependence of the measured insulation value (different even for the same type of real-OC) the inability to completely weed out the external random and non-random influence.

Obtained expressions for $D_{j(k)}$ make it possible to estimate the error (low value of real systematic error) after receiving the results of an increase in the calculated tangents at $\left.\mathrm{OC} \delta p_{j(k)}\right)$, assuming that the increase in real tangents corresponds to an increase of calculated $\left(\delta_{j(k)}{ }^{-}\right.$ $\left.\delta_{j(0)}=\delta p_{j(k)}-\delta_{j(0)}\right)$. Also expressions allow to assess the expected error in the determination of tangents calculated in the control system, making the assumption that the increase in real tangents at a few OC.

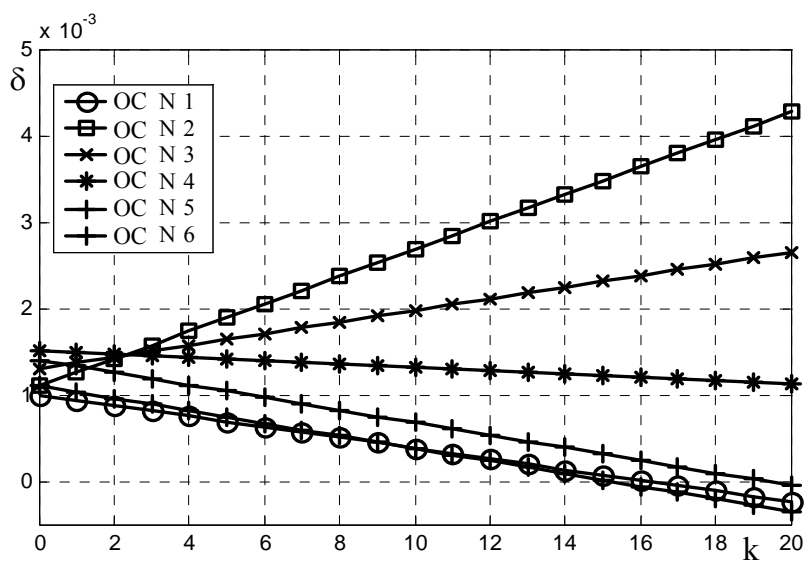

Fig. 3. Changes of calculated values of OC insulation loss tangents determined by method 2

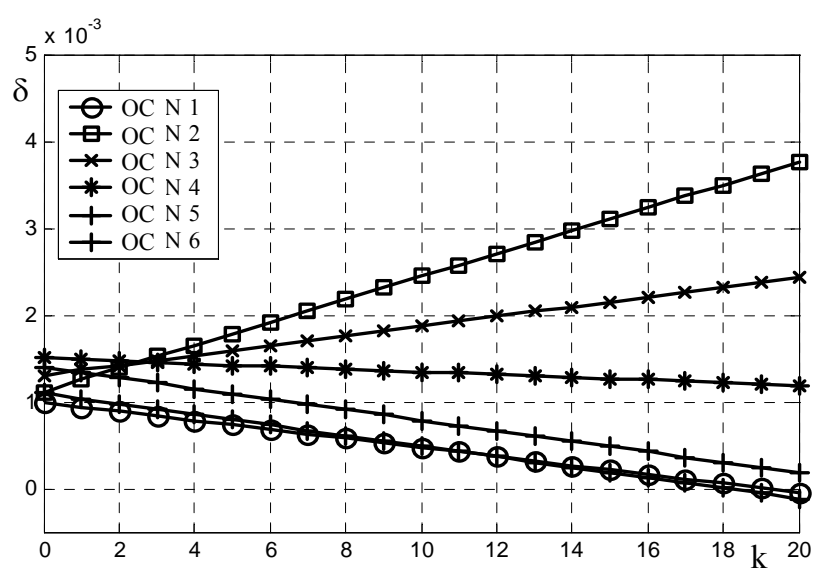

Fig. 4. Changes of calculated values of $\mathrm{OC}$ insulation loss tangents determined by method 3

As a result, it can be stated that the processing technique without isolating individual standard (methods 2 and 3) do not provide the advantages of precision in determining results but require substantially more measurements ( $N^{2}$ against $N$ in method 1$)$.

Methods improvement. Reducing methods' errors without isolation of the individual standard can be achieved by changing the order of calculation as follows. Averaging the results of measurements conducted in (7), (9) or (11) for determining the calculated tangent should be not OC in all, and on the selected smaller group of objects of volume $\mathrm{Nm}$. The composition of this «support» group defined in the previous $(k-1)$ time measurement step, by choosing OC with the smallest increase calculated relative to the tangent of the initial values: $\left(\delta p_{j(k-1)}-\delta_{j(0)}\right)$. With this selection of averaging excluded objects bearing potential danger of a large distortion of the calculated results, which reduces the expected error (total gain of tangents «support» of the group is less than the total increase). Thus, for example, the formula (7), (9) at the modified procedure will be written:

$$
\begin{aligned}
& \delta p_{i(k)}=-\frac{1}{N m-1} \sum_{j \neq i}^{N m} \Delta \delta_{i j(k)}+\delta_{i(0)}= \\
& =\frac{1}{N m-1} \sum_{j \neq i}^{N m}\left(-\partial \delta_{i j(k)}+\delta_{j(0)}\right),
\end{aligned}
$$

if the $i$-th OC is included in the selected group, if not - то in (13) instead $N m-1$ we should write $\mathrm{Nm}$.

Taking into account the low probability of simultaneous significant deterioration in many OC, «support» group may contain only a few objects smaller than the total number of OC. The greater part of the OC group, the less can change its composition.

To substantially reduce the number of measurements at each time step (from $\approx N^{2}$ to $\approx N$ ), one can determine the differential angle tangent of two OC in (7) and (9), (11), (13) by not a direct measurement but by calculation using measurements of the tangent with selection of an individual reference object: 


$$
\partial \delta_{i j(k)}=\partial \delta_{l j(k)}-\partial \delta_{l i(k)},
$$

where $l$ is the index of the selected reference object.

With this definition $\partial \delta_{i j(k)}$ we lose the opportunity to further test the results by comparing the results of the «direct» and «reverse» measurements $\left(\partial \delta_{i j(k)} \approx-\partial \delta p_{j i(k)}\right)$, proposed in $[2,7]$. Note that the specified test when necessary, without losing efficiency deviation of unsuccessful results it is possible to replace by the control deviations at the statistical processing of the results of repeated measurements with the selected reference.

Fig. 5 shows the variation of the calculated tangent defined by the formula (13), an improved method for modeling the situation described previously. When calculating the chosen «reference» group of 4 OC $(66 \%$ of total OC) having the lowest increase at the current time step, thus it was possible distorting effect of 2 and 3 OC on the results of the evaluation (changes to OC 1 and 4 continue to influence, causing error ). It can be seen that the calculated tangents better reproduce the values and trends of the real tangents than in methods 2 and 3. In contrast to the method 1 are controlled by all OC including standard.

Errors of methods without selection of the standard less at more controlled and $\mathrm{OK}$ with a smaller total change of tangents (a smaller increase in the values of real tangents, fewer $\mathrm{OC}$ to deterioration).

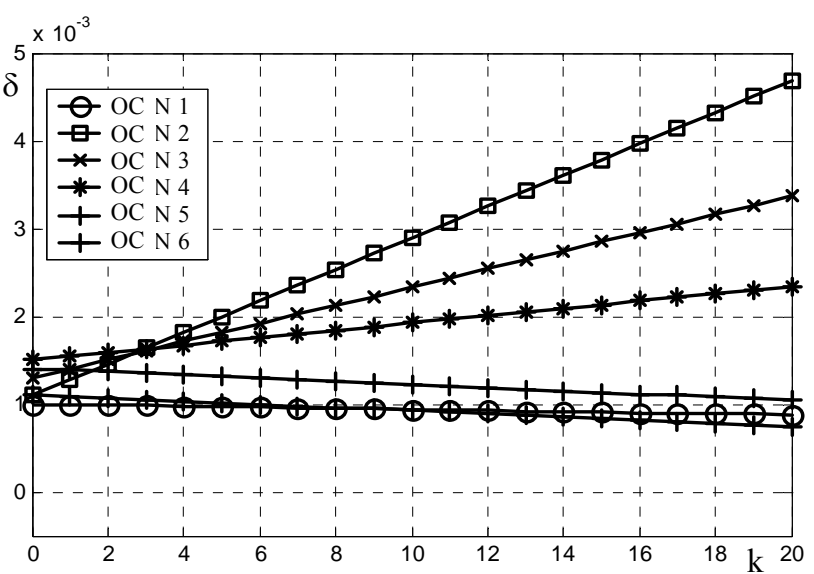

Fig. 5. Changes of calculated values of OC insulation loss tangents determined by improved method

Conclusions. A distinctive feature of methods without selection of reference is the impact of changes in loss tangent of one $\mathrm{OC}$ on the results of the assessment of other OC tangents leading to difficult to forecast the methodological errors that make it difficult to estimate the actual value of tangent and can cause errors in the assessment of controlled objects.

Appearances of at least one $\mathrm{OK}$ with severely deteriorating real parameters leads to a decrease in the estimated loss tangents of all OC that disguises the appearance of other disadvantaged OC with smaller deviations, and makes it difficult to identify such deviations for determining the developing insulation defect in the early stages.

The proposed improvement of methods for determining the estimated values of tangent with averaging over a dedicated «support» OC group allows reduce the negative impact of objects with deteriorating performance on the assessment of other OC tangents and reduce errors, while maintaining the ability to simultaneously control of all OC.

Utilization of the calculation method for determining the differential angle tangents of two $\mathrm{OC}$ on the results of measurements by the results of measurements using selected standard permits to significantly reduce the number of required measurements (till the value required in the traditional method with the selection of the standard).

In conclusion, conclusions are valid for both basic current transformers insulation control as well as for insulation monitoring of high-voltage bushings.

\section{REFERENCES}

1. Beliaev V.K., Borshchev P.I., Obodovskii V.D., Kanivetskii Iu.V., Bekhtev G.V., Bogdanov S.G., Masenko D.A., Dvoinykh V.P. Instrumentation and experience of monitoring of capacitor type insulation under operating voltage. Electrical networks and systems, 2012, no.4, pp. 68-72. (Rus).

2. Sakhno A.A. Measurement algorithm of the dielectric loss tangent of basic insulation of current transformers and bushings $330-750 \mathrm{kV}$ under monitoring under a working voltage. Electrical engineering \& electromechanics, 2010, no.2, pp. 54-56. (Rus.) doi: 10.20998/2074-272X.2010.2.14.

3. SOU-H MPE 40.1.46.301:2006. Perevirka izoliatsii transformatoriv strumu 330-750 kV pid napruhoiu. Metodychni vkazivky [Standard of organization H MPE 40.1.46.301:2006. Check of insulation of current transformer $330-750 \mathrm{kV}$ under voltage. Methodical instructions]. Kyiv, Minpalyvenerho Publ., 2006. (Ukr).

4. Stognii B.S., Pilipenko Iu.V., Sopel' M.F., Tutik V.L. Hardware-software monitoring complex of insulation of current transformers and high-voltage bushings of power transformers. Works of the Institute of Electrodynamics of the National Academy of Sciences of Ukraine, 2010, is.26, pp. 38-45. (Rus).

5. Shinkarenko G.V., Onishchenko V.A., Ornatskii O.A. Technologies of measurement of insulation parameters of oilfilled transformers $330-750 \mathrm{kV}$ under operating voltage. Electrical networks and systems, 2012, no.3, pp. 67-71. (Rus.)

6. Svi P.M. Metody i sredstva diagnostiki oborudovaniia vysokogo napriazheniia [Methods and diagnostic tools of the equipment of a high voltage]. Moscow, Energoatomizdat Publ., 1992. 240 p. (Rus).

7. Beliaev V.K., Panenko H.N. Features of definition methods of insulation parameters in the monitoring systems of current transformers under working voltage. Bulletin of Kharkiv Petro Vasylenko National Technical University of Agriculture. Series: Technical science, 2014, no.153, pp. 122-124 (Rus). 
8. Beliaev V.K., Obodovskii V.D., Panenko H.N. The analysis of sensitivity of diagnostic models for the monitoring of a transformer windings condition under load. Tekhnichna elektrodynamika, 2013, no.4, pp. 81-87. (Rus).

Received 11.06.2016
V.K. Beliaev ${ }^{1}$, Candidate of Technical Science, Associate Professor,

H.N. Panenko ${ }^{2}$, Postgraduate Student,

${ }^{1}$ Scientific Industrial Enterprise «OST»,

33-D, Bulvarno-Kudryavskaya Str., Kyiv, 01054, Ukraine. e-mail: bel_vk@ua.fm

${ }^{2}$ National Technical University of Ukraine «Kyiv Polytechnic Institute»,

37, Prospect Peremohy, Kyiv-56, 03056, Ukraine.

How to cite this article:

Beliaev V.K., Panenko H.N. Determination of insulation parameters of current transformers at multiple measurements in monitoring systems under working voltage. Electrical engineering \& electromechanics, 2016, no.5, pp. 40-46. doi: 10.20998/2074-272X.2016.5.06. 